\title{
Chernobyl-Touched Children: E and A Hypovitaminoses-Related Mechanisms and Prevention of Radiogenic Mutagenicity and Teratogenicity
}

\author{
Eugene Neyfakh ${ }^{1 *}$, Aigule Alimbekova ${ }^{1}$ and Igor Suskov ${ }^{2}$
}

${ }^{1}$ Semenov N, Institute of Chemical Physics, Russian Academy of Sciences, Moscow, Russia

${ }^{2}$ Vavilov N, Institute of General Genetics, Russian Academy of Sciences, Moscow, Russia

\begin{abstract}
Children and their mothers affected by the Chernobyl catastrophy suffer with toxic lipoperoxic stress coupled with hypovitaminoses $\mathrm{A}$ and $\mathrm{E}$. The relation of these primary biochemical disturbances to notorious somatic consequences on mutagenic and teratogenic levels caused by chronic low-dose radiation of children from radiopolluted regions had been investigated. Rational approaches to prevent or to treat indicated pathologies were developed either. Vitamins $A$ and $E$ blood levels for newborns from radio polluted regions were near a third of normal ones. Simultaneous increasing of lipo-peroxidation for studied children had tendency to correlate with their body stigmation. Radiogenic indices of mutagenicity and teratogenicity showed dramatic increases along with fall of the essential antioxidants. The peroral poly vitamin therapy of women at the beginning of $1^{\text {st }}$ trimester pregnancy prevented or corrected evaluated abnormalities for their newborns. Vitamin-protected children had normal ranges for studied indices. The combination of radiogenic biochemical deviations is supposed to be the primary molecular mechanisms for children restorable somatic hyper mutations and irreversible body stigmations but preventable by essential antioxidants via their mothers at early pregnancy.
\end{abstract}

Keywords: Bioantioxidants; Lipoperoxidation; Radiogenic; Mutagenicity; Teratogenicity; Children

\begin{abstract}
Abbreviations: $\quad \mathrm{vA}$-vitamin $\mathrm{A}$; $\mathrm{vE}$-vitamin $\mathrm{E}$; BAO-essential bioantioxidants; LPC-lipoperoxic cascade; D-total radioloads; Daantenatal radiodoses; Dm-maternal radiodoses; MEAN-minor embryogenic anomalies of newborns; LCM-lymphocytic chromosomes mutagenesis.
\end{abstract}

\section{Introduction}

Enhanced levels of minor embryogenic anomalies for newborns (MEAN, so-called stigmae) and Lymphocytic Chromosomes Mutagenesis (LCM) are phenotypic manifestations of somatic hypermutagenesis and organogenesis disturbances. These pathologies are regarded to be coupled with elevated risks of tumors, immune incompetence and accelerated aging; irreversible MEAN have evident negative psychologic effects also. LCM and MEAN are very sensitive to ionizing irradiation, especially during pregnancy and infancy. Into radioisotopes-polluted regions as a result of the Chernobyl catastrophy-1986, enhanced LCM levels [1-3] and / or various congenital malformations [4] for newborns and children were observed. The molecular mechanisms of these phenomena were investigated and discussed by us earlier $[5,6]$ but they are still obscure.

The major aims of the recent investigation were the following:

1. To ascertain roles of deficits in essential lipid bio-factors as vitamins $\mathrm{A}(\mathbf{v A})$ and $\mathrm{E}(\mathbf{v E})$, as interrelated enhanced lipoperoxidation for radiogenic somatic hyper-mutagenesis and body stigmations of newborns and children who were residents of Chernobyl-radio polluted regions.

2. To check for embedding preventive (for MEAN) and therapeutic (for LCM) capabilities of cheap multivitamins for early pregnant women, newborns and children to radiogenic enhancement of stigmation, as to somatic hyper mutagenesis.

\section{Materials and Methods}

\section{Contingents and radiodosimetry}

Children (from newborns up to 3 yrs old inclusive, $n=217$ ) and some of their mothers were investigated. They were residents of radio polluted regions: from Mtsensk (I, $\mathrm{n}=73$ ) and Bolkhov (II, $\mathrm{n}=47$ ), towns of t. Orel district, Russia; and from Chechersk town (III, $\mathrm{n}=$ 97) of t. Gomel district, Belaruss'. Surface mosaic radio pollution for I, II and III were 1-5, 5-15 and $15-40 \mathrm{Ci} / \mathrm{km}^{2}$ by ${ }^{137}$-Cs, respectively. I and II are located between $40 \mathrm{~km}$ interval and into a joint radioactive spot; III is located $190 \mathrm{~km} \mathrm{~N}$.-E. from the Chernobyl APS. Individual rates of external summary $\gamma$-irradiation for children (D), antenatally for fetuses (Da) and their mothers (Dm) were determined with the DRG-01T counter, portable cumulative thermo luminescence detectors and the Harshaw TLD 3500 detector; accumulated radioloads were reconstructed as described in [5-7]. As D and Dm are strongly correlated with internal radiation loads for studied districts, evaluated effects are result of general irradiation due to radiogenic mechanisms for studied biochemical and somatic deviations [1-4,8-10].

\section{Study design}

Formation of comparable contingents, the therapy and all results were performed by the double-blind approach for involved investigators. Contingents were age and place-matched, data of persons without polyvitamin treatment were paired-compared ones during computation. Criteria of normalization follow from children normal age diapasons for studied blood vitamins and LPC $[9,10]$.

\section{Biochemical analysis}

Plasma samples of morning fasting blood or of cord newborns'

*Corresponding author: Eugene Neyfakh, Inst. of Chemical Physics RAS, Moscow, Russia, Tel: +495-9397475; Fax: +499-1374101; E-mail: ney@land.ru

Received November 16, 2013; Accepted December 24, 2013; Published December 30, 2013

Citation: Neyfakh E, Alimbekova A, Suskov I (2013) Chernobyl-Touched Children: $\mathrm{E}$ and A Hypovitaminoses-Related Mechanisms and Prevention of Radiogenic Mutagenicity and Teratogenicity. Vitam Miner 3: 117

Copyright: (c) 2013 Neyfakh E, et al. This is an open-access article distributed under the terms of the Creative Commons Attribution License, which permits unrestricted use, distribution, and reproduction in any medium, provided the original author and source are credited. 
blood withdrawals were fixed by dry ice and then transported into Dewar vessels to the Moscow IBCP lab. Lipids were extracted quantitatively from samples by vortexing in ethanol: hexan $(1: 2, \mathrm{v} / \mathrm{v})$ (Uvasol, Merck, Germany) with $1 \%$ ascorbate at $\mathrm{pH} 4,5$ under purified argon. Free tocopherol were determined at $295 / 325 \mathrm{~nm}$ and retinol at $330 / 470 \mathrm{~nm}$ fluorometrically (spectrofluorometer 850, Hitachi, Japan) with corresponding their standards (Serva, Austria) [11,12]. LPC catabolites as diene conjugates and ketodienes were determined Spectrophotometrically by their extinction coefficients $[13,14]$. Absence of artifacts was proved by sets of controls at all assay steps.

\section{Mutagenicity and teratogenicity}

Blood cells, pre-separated from the same samples as in 2.3 before plasma freezing, were investigated for LCM indices in the IGG lab, Moscow. Microscopic slides were prepared for chromosomal analysis according to the standard procedure [15]. Preparations stained with the fluorescence plus Giemsa technique were analyzed for various mutations according to [3] and LCM indices were expressed as mutations frequencies. Levels of stigmations disposed in head area mainly were evaluated visually according to their nomenclature [16] and supplied by pediatricians of local maternity hospitals. MEAN indices are defined as total number of minor anomalies per the newborn. Up to 5 MEAN per a person are accepted in neonatology into their normal diapason as somatic diversity [16]. Radiogenic stigmation or radiogenic mutagenesis is defined as ratio of MEAN or LCM indices to children or their mothers' radioloads in cSv. Values of slopes for graphs of LCM / cSv or MEAN / cSv were used as radio-sensitivity parameters for studied children.

\section{Multivitamin therapy}

Women $(\mathrm{n}=22)$ from II in $1^{\text {st }}$ and $7^{\text {th }}$ months of pregnancy obtained poly vitamins "Unicap Jr." (USA) containing essential bioantioxidants for 3 weeks dayly : vE-20 mg, vA-3 mg, vC-120 mg. Their infants obtained the half-dosed curses with meal. Composition of vitamins per a tablets contains: vitamins A (5000 IU), E (15 IU), B1 (1.7 mg), B6 (2 mg), B12 (6 mcg), C (60 mg), D (400 IU), folic acid (400 mcg) and niacine $(20 \mathrm{mg})$. Children from I and III towns and their mothers (n $=55$ ) obtained no vitamins and were used to evaluate radiation effects and to compare with vitamin-protected contingents.

\section{Mathematical analysis}

Statistical probabilities of linear regressions for paired data were determined by the Wilcoxon test with $95 \%$ confidence. To evaluate hyperbolic-like functions 3 rd derivatives for graphs of regressions were used.

\section{Results}

\section{Essential antioxidants}

Figure 1 shows that levels of $\mathrm{vA}$ and $\mathrm{vE}$ for newborns decrease along with increasing their antenatal and / or maternal radioloads. If women in early pregnancy had no obtained the vitamin therapy,

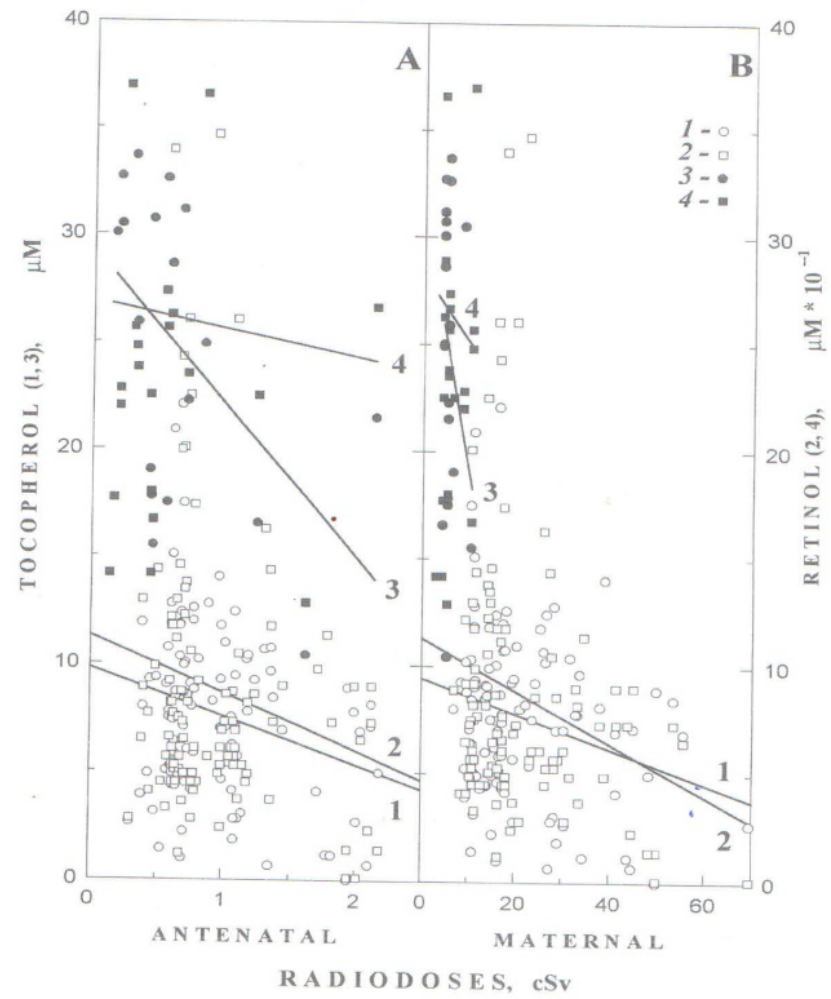

1-tocopherol, Chechersk : (A) $r=-0.251, p=0.012, n=99 ;(B) r=-0.250, p=0.014, n=97$.

2-retinol, Chechersk : (A) $r=-0.261, p=0.034, n=96$; $(B) r=-0.241, p=0.017, n=97$.

3-tocopherol, Bolkhov: (A) $r=-0.521, p=0.027, n=18$; (B) $r=-0.291, p=0.226, n=19$.

4-retinol, Bolkhov : (A) and (B)-insignificant correlations

BAO-therapy: Bolkhov;

Without the therapy: Chechersk.

Figure 1: Da and Dm radioloads influence on development of children hypovitaminoses $A$ and $E$. 
their posterity even with the least radioloads (obtained from external sources or via their mothers) were born with about a third of normal $\mathrm{vA}$ and $\mathrm{vE}$ levels, while antenatal $2.5 \mathrm{cSv}$ or maternal $70 \mathrm{cSv}$ radio doses decrease their hypovitaminosic levels further down in 2-3 times to near $15 \%$ of their mean norms (Figure 1; A, B: 1, 2). A considerable part of the unprotected contingents was born with profound hypo- or avitaminoses A and E. Although, if chronically irradiated pregnant women, residents of radio polluted regions had obtained early BAO therapy, their newborns had normal BAO levels, which also decreased however while rising of both radioloads. While newborns of vitaminsuntreated mothers showed negative significant correlations " $\mathrm{Da}$ or $\mathrm{Dm}$ vs BAO" correlations "Dm vs newborns" BAO became insignificant if mothers had been BAO-treated. In spite of about 30-fold weaker influence on children BAO levels of maternal radioloads than of equal antenatal ones, both sources of radioloads result in similar and the most harmful consequences for posterity due to much higher accumulated radioloads of residential mothers [10].

\section{Mutagenicity and teratogenicity}

The increases in level of lipoperoxic metabolites (diene conjugates, DC, and ketodienes, KD) for unprotected newborns correlate significantly with their stigmation levels (Figure 2) Effects of vA and vE levels on the values of radiogenic mutagenesis and stigmation indices show Figure 3 and Figure 4. For Bolkhov newborns protected by BAO therapy up to vA and vE normal levels, the values of all radiogenic indices lie within the scatter of their norms; nevertheless they gradually increase several times along with radiogenic BAO declines (Figure $1,3,4)$. Indices of radiogenic mutagenesis show significant inverse linear correlations with vA, and insignificant one with vE. For BAOunprotected newborns, growth of $\mathrm{vA}$ and $\mathrm{vE}$ deficits have resulted in dramatic increase of all radiogenic somatic indices. Their radiogenic stigmation indices also correlated significantly with retinol, but not with tocopherol deficits. For these, the slopes of the graphs " radiogenic mutations vs $\mathrm{BAO}$ " exceeded that for $\mathrm{BAO}$-protected individuals 13 to 22 -fold and 32 to 80 -fold for exhausting of $\mathrm{vA}$ and $\mathrm{vE}$, respectively. For children without BAO-therapy, the only graph "\% chromosome single breakage / cSv "plotted vs vE revealed zero slope (Figure $4 \mathrm{C}$ ), meanwhile, its values exceeded 12-fold of normal one. However, the index was depended on vA and sloped significantly for unprotected children (Figure $3 \mathrm{C}$ ). As indicators of radiolability, the slopes of radiogenic stigmation graphs for unprotected children exceeded the same for BAO-protected ones in 13 and 6 times during $\mathrm{vA}$ and $\mathrm{vE}$ decreasing, correspondingly. As a result of $\mathrm{Da}$ and / or Dm actions on comparable united contingents from I and II, the indices of radiogenic stigmation grow according to function close to hyperbola when and after vA and vE levels fall lower than $1.7 \mu \mathrm{M}$ and $17 \mu \mathrm{M}$, respectively (Figure 5). The same is true for the majority of radiogenic mutagenesis indices (not presented, Figure 3, 4A, 4B, 4C).

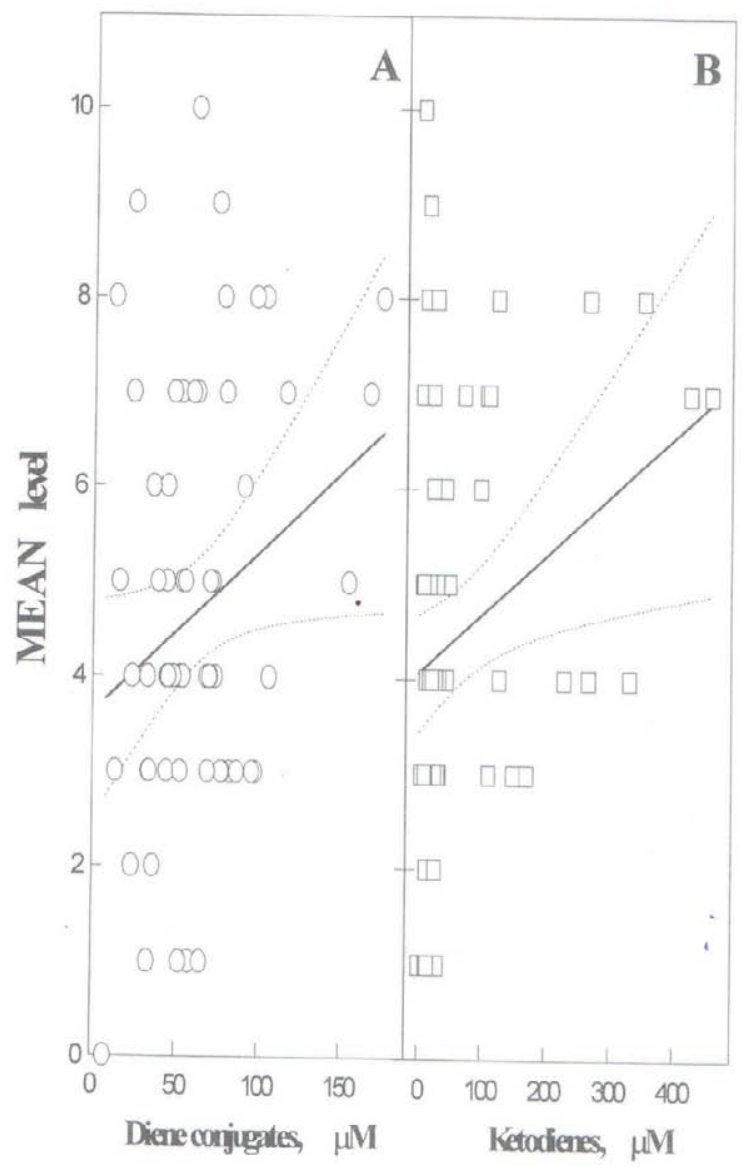

A: $r=0.260, p=0.04, n=61$

B: $r=0.300, p=0.01, n=64$

Figure 2: Tendencies or positive correlations MEAN with levels of lipoperoxic catabolites for newborns of untreated mothers, Chechersk. 


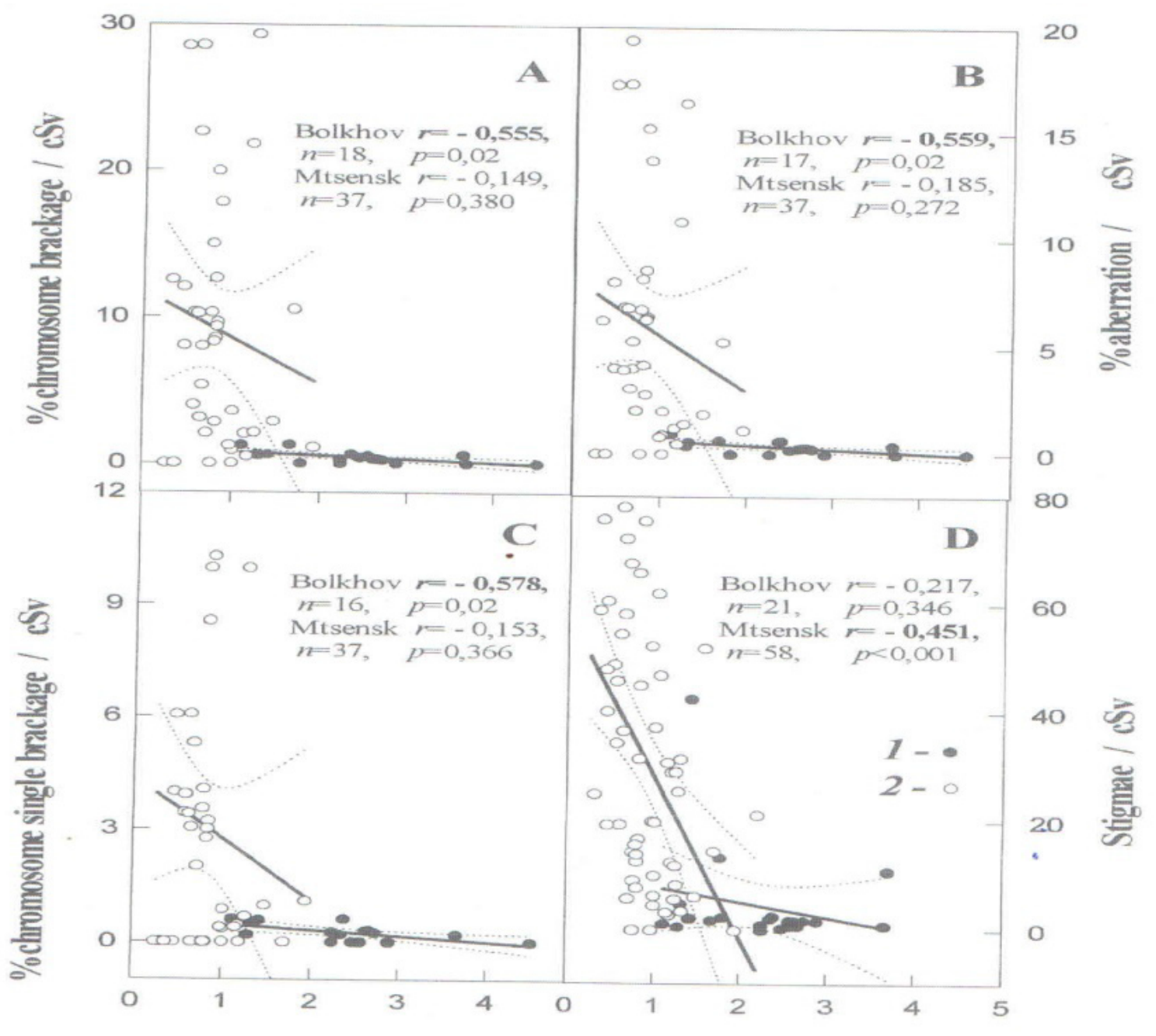

R E T I N I, $\mu$ M

1: BAO-therapy (Bolkhov)

2: without therapy (Mtsensk)

Figure 3: Normalization of children radiogenic retinol-related mutagenesis and stigmations by maternal polyvitamin therapy.

\section{Discussion}

We have discovered and proved the novel radiogenic syndrome for children and adults affected with Chernobyl catastrophy: the lipoperoxic stress coupled with hypovitaminoses A and E $[9,10,17-$ 19]. For newborns to preschool children, residents of radiopolluted areas, a lot of various significant correlations of the type " raiodoses vs effects " were found, owing to the highest radiolability of the contingents studied, high sensitivity of metabolic test systems used, and prevalence of this syndrome in the area of former USSR. Similar health deviations are waited at similar radiogenic accidents (into the Fukushima APS accident-touched area, as an example). Therefore, we investigated the possibility if the radiogenic biochemical disturbances found could affect the levels of somatic chromosomal mutagenesis and minor embryogenic anomalies $[17 \mathrm{~b}, 18 \mathrm{~b}]$. All deviations found resulted not only from registered external irradiation of children, but also from internal one, as it is follows from high correlation of external with internal radionuclidic loads $(\mathrm{r}=0.94, \mathrm{p}<0.001)$ for studied residents (Figure 6) estimated from $[8,20]$.
We have asserted previously that direct correlations of maternal radioloads with long-term biochemical deviations of their children resulted from effective uptake of maternal incorporated radionuclides (in proportion values to $\mathrm{Dm}$ ) by a fetus via cord blood and then by an infant via milk during suckling and their long retaining [10]. Chronic low-dose ionizing irradiation, initiating free radical lipoperoxic cascade of polyunsaturated lipids (with effective participation of body biocatalysts and oxygen), in order to raise normal steady levels of LPC catabolites by 1-2 orders of magnitude (Figure 2) $[9,19]$ and their possible modifications with coupled decrease of BAO levels down to deep hypovitaminoses (Figure 1) [10].

There are many evidences for toxic, carcinogenic, mutagenic and teratogenic properties of enhanced levels of lipoperoxic catabolites and products, which are capable to act as independent stressoric biofactors amplifying the direct irradiation effects. Previously, one of us (by EN) had found that highly sensitive developing quail embryos die with teratogenic injuries after injections in ovo of lipids from organs of LPC-stressed rodents [12,21]. The effects were lipoperoxide-dependent 


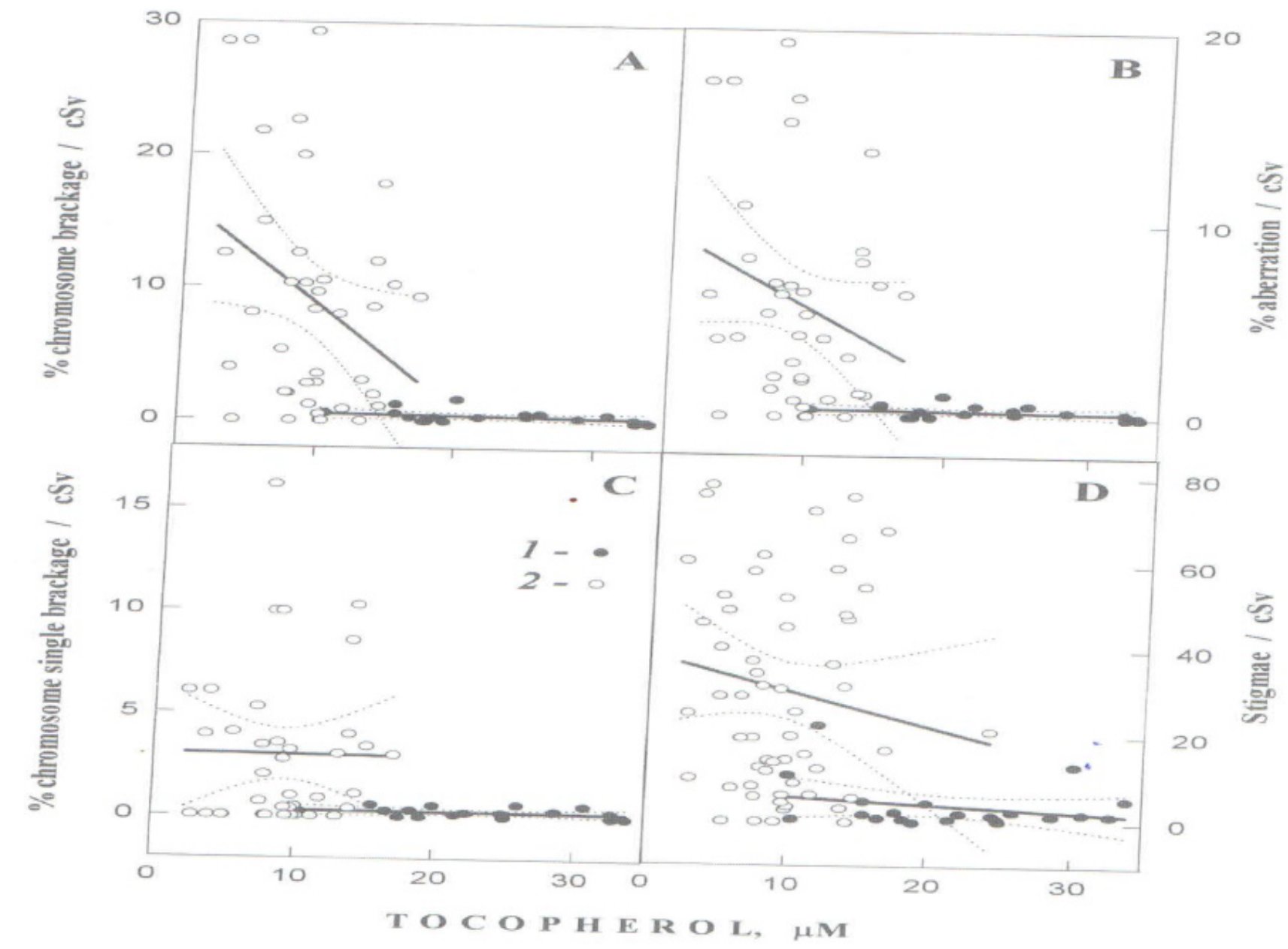

1: BAO-therapy (Bolkhov)

2: without therapy (Mtsensk).

Figure 4: Normalization of children radiogenic tocopherol-related mutagenesis and stigmations by maternal polyvitamin therapy.

and were eliminated by preliminary reduction of bio-accumulated LPC catabolites from lipids.

Enhanced levels of LPC catabolites have capable to bring about various chromosomal mutations [22]. Children of Chernobyl suffer with LPC-stress and deficits in retinol and tocopherol both due to their enhanced expenditure as BAO $[9,10,17 \mathrm{~b}, 18 \mathrm{~b}]$.There are linear correlations between declines of these BAO levels and increments for LCM, MEAN, MEAN / Da and MEAN / Dm indices. However, for badly lowered vA and vE levels in unprotected children, just here hyperboliclike rises proceed for all indices (Figure 5). Radiogenic indices show inverse correlations with $\mathrm{BAO}$ levels (Figure 3,4). For example, if retinol level falls to near $0.5 \mathrm{M}$, that is typically for studied unprotected large contingents (Figure 1), the values of the indices exceed 28-46-fold the normal ones (Figure 3,5). The effect of simultaneous vE deficit on enhancing the radiogenic mutagenesis were 3-4 times higher than that of $\mathrm{vA}$, but retinol deficit enhanced radiogenic stigmation twice more effective than that of tocopherol, that is follows from comparison of slopes for graphs of radiogenic indices as radio-sensitivity parameters (see Results, 3.2). It is possible that the specificity of these biofactors manifested on the molecular level just here. This specificity may be also investigated with the aid of revealed peculiarities of single chromosomal breaks during development of radiogenic deficits of vA (Figure 3) and vE (Figure 4).

It is possible that electrophilic LPC catabolites, accumulated with development of BAO deficits during irradiation, and their free radicals form strong adducts with chrosomal DNA and cause the observed disturbances of mutagenesis and organogenesis, so amplifying the direct radiation damages either. In that case, the combination of both pathogenic components of the evaluated primary Chernobyl biochemical syndrome may be just the proper radiogenic molecular mechanisms which have caused secondary somatic disturbances of children on mutagenic and / or teratogenic levels. Irreversible MEAN follow radiogenic vA and vE deficits and / or LPC stress, but not vice versa, due to the preventive effects evaluated for early BAO-therapy (Figure 3-5). Frequency of congenital malformations in newborns and human embryos has increased in radio contaminated Belaruss' areas following the catastrophy [4,23]. For the studied Gomel' district germline mutations either at human mini satellite loci were twice as high as the controls and were correlated with ${ }^{137}$-Cs surface contaminations [2]. It is quite possible that chronic low-dose irradiation are more effective in mutation and stigmation inductions than equal doses of acute irradiation at higher rate $[2,23,24]$. Evaluated LPC and BAO 


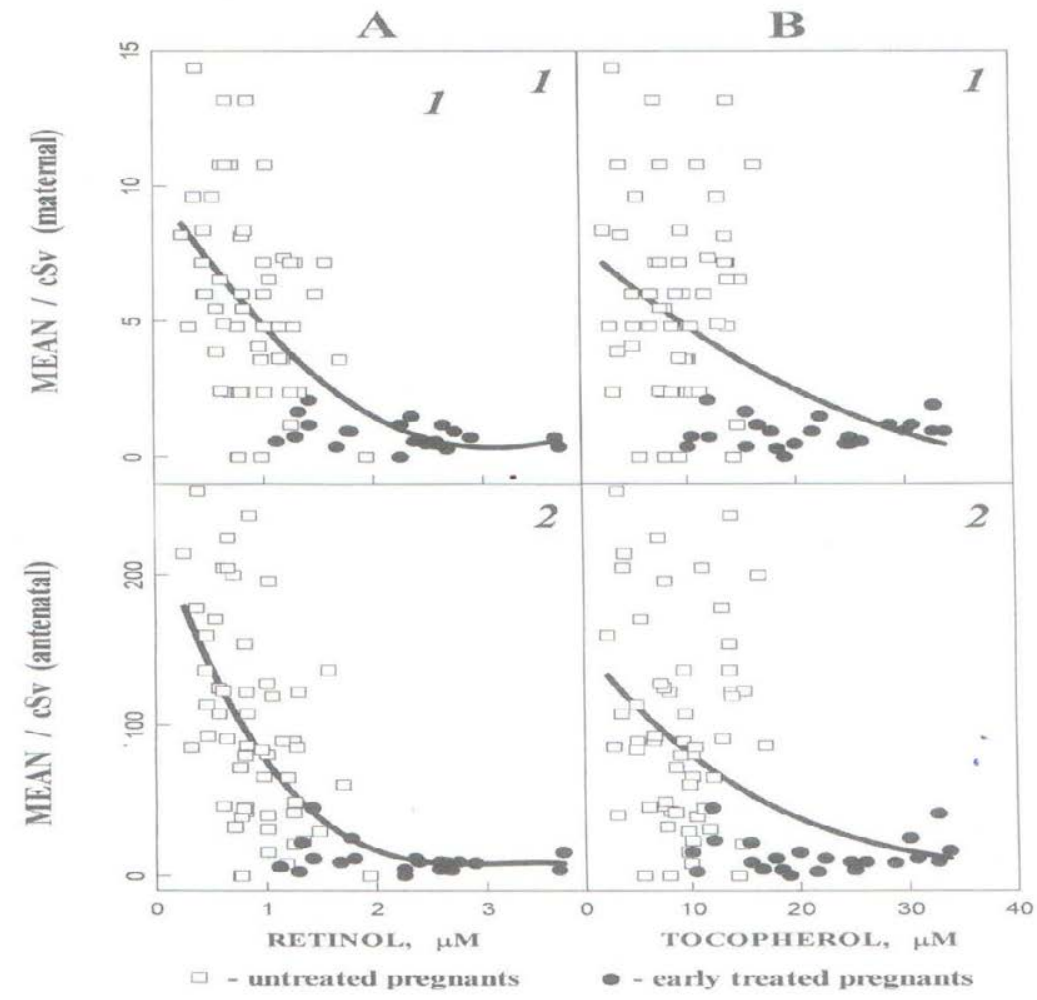

1-stigmations produced by maternal radioloads

(A)-retinol (B)-tocopherol

2-stigmations produced by antenatal radioloads

Figure 5: A and E hypovitaminoses-related hyperbolic-like increases of radiogenic body stigmations.

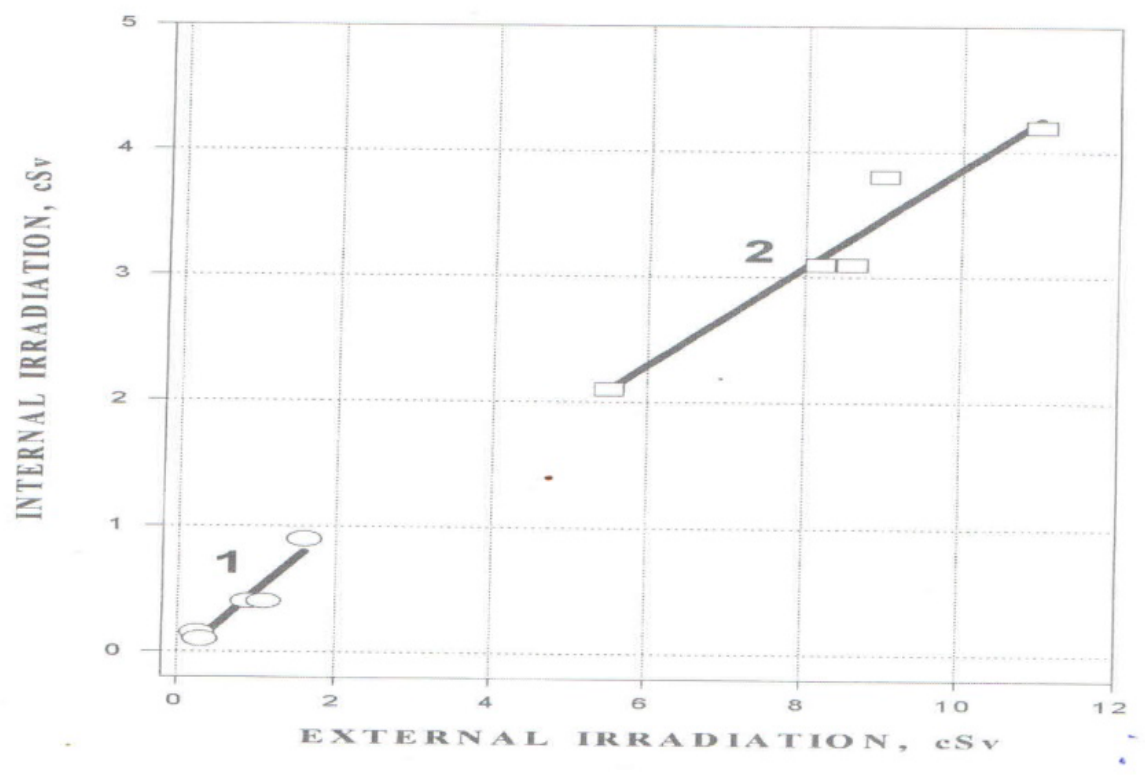

Regions: Gomel' and Mogilev districts, as Bryansk district

1-minimal doses $(Y=-0.10+0.57 x ; r=0.940, p<0.001, n=5)$

2-maximal doses $(Y=-0.07+0.39 x, r=0.938, p<0.01, n=5)$.

Estimated from reconstructed radiodoses and data of post mortem skeletons radioactivity (internal radioloads) and radiopollutions (external $\mathrm{Y}$-irradiation) for contingents of studied districts $[8,20]$

Figure 6: Correlations external with internal radioloads for Chernobyl-touched populations. 
Citation: Neyfakh E, Alimbekova A, Suskov I (2013) Chernobyl-Touched Children: E and A Hypovitaminoses-Related Mechanisms and Prevention of Radiogenic Mutagenicity and Teratogenicity. Vitam Miner 3: 117.

deviations in blood $[9,10]$ are supposed to be so-called "clastogenic factors" in the plasma of children exposed at Chernobyl [15].

It is of high practical importance that all revealed children deviations were curable or preventable, as in the case of adequate $\mathrm{BAO}$ treatment in early pregnancy all studied records for newborns became in normal limits (Figure 1 (3), Figure 3, 4,1). Peroral polyvitamin therapy of pregnant women (or radiation-touched women of fertile ages beforehand) and then of their children, who were residents of radio polluted regions, has several indispensable benefits : 1) high efficiency against studied radiogenic pathologies, 2) absence of contraindications at adequate usage, 3 ) a relative low price combined with simplicity for the therapy.

These decisive advantages determine the approach for victims suffering from radiogenic health consequences caused by Chernobyltype accidents as the method of choice [9,10,18,19,25-27]. Polyvitamin therapy will be useful also for any other contingents affected with chronic irradiations, including from Fukushima-touched regions, because vitamins uptake from meal being adequate under usual conditions, is no able to compensate accelerated $\mathrm{BAO}$ expenditure as a result of prevention of radiogenic-initiated chain-branched LPC in an being irradiated organism $[17,28,29]$.

\section{Acknowledgements}

The Project based on mutual data-exchange principals for participants was supplied by Institute of Chemical Physics, Institute of Biochemical Physics, and Institute of General Genetics, Moscow, all of the Russian Academy of Sciences. We are greatly thankful Dr. A.A. Kriminsky (Institute of Medical Biophysics, Moscow) for technical radiodosimetric data, Dr. Inna Tsibulskaya (Research Center of Perinatology, Obstetrics and Gynecology, Moscow) for newborns' data and Dr. V.A. Andreev for fruitful discussions and help.

\section{Conflicts of Interests}

None to declare

\section{References}

1. Shevchenko VA, Snigiryova GP (1996) Cytogenetic effects of the action of ionizing radiation on human populations. In: Consequences of the Chernobyl catastrophe: human health. Burlakova EB (ed) Moscow, Russia

2. Dubrova YE, Nesterov VN, Krouchinsky NG, Ostapenko VA, Neumann R, et al (1996) Human minisatellite mutation rate after the Chernobyl accident. Nature 380: 683-686.

3. Lazutka JR (1996) Chromosome aberrations and rogue cells in lymphocytes of Chernobyl clean-up workers. Mutat Res 350: 315-329.

4. Laziuk GI, Kirillova IA, Dubrova luE, Novikova IV (1994) [Incidence of developmental defects in human embryos in the territory of Byelarus after the accident at the Chernobyl nuclear power station]. Genetika 30: 1268-1273.

5. Wallstram E, Wahni T (1991) Measurements on individual doses from externa radiation in the Bryansk region of the RSFSR. Report Statens Institute for Stralhygiene. National Institute of Radiation Hygiene. Norway.

6. Minenko VF, Drozdovich VV, Tretyakevich SS (1996) Methodological approaches to calculation of annual effective dose for the population of Beloruss'. Bull Radiation and risk 7: 246-252

7. Balonov MI (1996) Exposure of the population of Russian Federation as a result of the Chernobyl accident. Ibid., 39-71.

8. Khramchenkova OM (1996) Certain features of accumulation of Strontium-90 in human body and its role in the formation of internal irradiation doses under conditions of Chernobyl accident, PhD Dissertation, Obninsk, Moscow, Russia

9. Neyfakh EA, Alimbekova AI, Ivanenko GF (1998) Radiation-induced lipoperoxidative stress in children coupled with deficit of essential antioxidants. Biochemistry (Mosc) 63: 977-987.

10. Neyfakh EA, Alimbekova AI, Ivanenko GF (1998) Vitamin E and A deficiencies in children correlate with Chernobyl radiation loads of their mothers. Biochemistry (Mosc) 63: 1138-1143

11. Tompson JN, Erdody P, Maxwell WB (1973) Simultaneous fluorimetric determination of vitamins $A$ and $E$ in human serum and plasma. Biochem Med 8: 403-414.

12. Neyfakh EA (1977) The high-sensitive method for investigation of lipoperoxide toxicity. Voprosy med khimii 23: 131-136.

13. Pompella A, Maellaro E, Casini AF, Ferrali M, Ciccoli L, et al. (1987) Measurement of lipid peroxidation in vivo: a comparison of different procedures. Lipids 22: 206-211.

14. Klein RA (1970) The detection of oxidation in liposome preparations. Biochim Biophys Acta 210: 486-489.

15. Emerit I, Quastel M, Goldsmith J, Merkin L, Levy A, et al. (1997) Clastogenic factors in the plasma of children exposed at Chernobyl. Mutat Res 373: 47-54.

16. Tabolin V, Shabalova N (1984) The list of oft-reccuring stigmae, In: Reference book of neonatalogy Medizina.

17. a) Neyfakh EA, Alimbekova A (1998) Symptoms of radiogenic free radical chain-branched process for Chernobyl-touched persons. In: Conf. "The role of free radicals in health and diseases", Abstracts p. 189; Jerusalem-Amman.

b) Alimbekova A, Ivanenko G, Neyfakh E (1998) Lipoperoxic-antioxidants pathologies with mutagenesis acceleration for children from Chernobylradiopolluted regions.

18. a) Alimbekova A, Ivanenko G, Neyfakh E (1998) Rational prevention of radiogenic minor embryogenic anomalies for Chernobyl low-dose irradiated newborns. In: 3rd Int.Congress on vitamins and related biofactors, Germany.

b) Neyfakh E and Alimbekova A (1998) Radiogenic hyporetinolemia has multiplied dramatically teratogenicity of irradiation for Chernobyl-touched newborns.

19. Neyfakh EA, Ivanenko GF. Chernobyl victims: deficits of essential antioxidants and lipoperoxide pathway disturbance as remote consequences. In: I Int. Congress "Vitamins and biofactors in life sciences" Japan.

20. Golovko OV, IzhevskiÄ PV (1996) [Study of reproductive behavior in Russian and Byelarus populations exposed to ionizing radiation as a consequence of the Chernobyl AES accident]. Radiats Biol Radioecol 36: 3-8.

21. NeÄfakh EA (1976) [Toxicity of lipids from the organs of tumor-bearing animals] Dokl Akad Nauk SSSR 230: 1470-1473.

22. Colin C, Narbonne JF, Migaud ML, Grolier P, Cassand P, et al. (1991) Lipid peroxidation and benzo[a]pyrene activation to mutagenic metabolites: in vivo influence of vitamins $A, E$ and $C$ and glutathione in both dietary vitamin $A$ sufficiency and deficiency. Mutat Res 246: 159-168.

23. Yablokov AV (1996) The Chernobyl Mythology, Environmental Policy Review (Jerusalem)10: 5-30.

24. Crompton NE, Barth B, Kiefer J (1990) Inverse dose-rate effect for the induction of 6 -thioguanine-resistant mutants in Chinese hamster V79-S cells by $60 \mathrm{Co}$ gamma rays. Radiat Res 124: 300-308.

25. Schmitz-Feuerhake I, Schmidt M (1998) In: Proceedings "Radiation exposures by nuclear facilities. Evidence of the impact on health", Ges. Fur Strahlenschutz, Berlin-Bremen, Germany.

26. Schmitz-Feuerhake I (1997) Leukemia in the proximity of a German boilingwater nuclear reactor: evidence of population exposure by chromosome studies and enviromental radioactivity. Env Health Persp 105: 1499-1504.

27. Neyfakh EA (2009) High pathogenic loading of children caused by "low-doses" of chronic radiation. In: Proceed. Int. Conf. "Biological consequences of lowdose radiation contamination in the environment".

28. Neyfakh EA (2013) "Low doses" of technogenic radiation are responsible fo significant radiopathogenic loads of children. In : Int. Conference "Radiation, ecology and technosphere" 2013, 110-113.

29. Fukushima Daiichi Nuclear Disaster. Wikipedia, 2012 